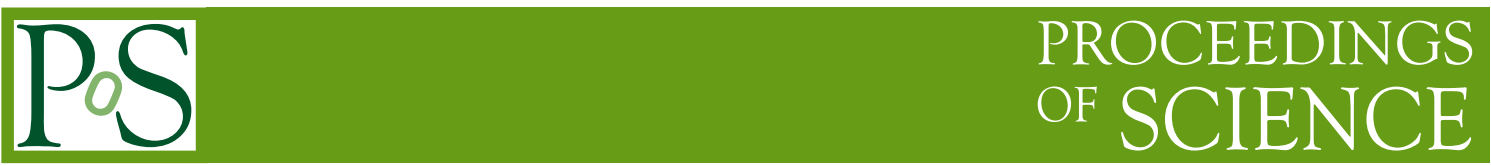

\title{
Progress in the Construction of the MICE Cooling Channel and First Measurements
}

\author{
A. Dobbs * \\ Imperial College London \\ E-mail: a.dobbs07@imperial.ac.uk
}

\section{Rayner}

Univeristy of Geneva

E-mail: mark.raynerecern.ch

\section{for the MICE Collaboration ${ }^{\dagger}$}

The Muon Ionization Cooling Experiment (MICE) is a strategic R\&D project intending to demonstrate the only practical solution to prepare high brilliance beams necessary for a Neutrino Factory or Muon Collider. MICE is under development at the Rutherford Appleton Laboratory, UK. It comprises a dedicated beam line to generate a range of input emittance and momentum, with time-of-flight and Cherenkov detectors to ensure a pure muon beam. The emittance of the incoming beam is measured in the upstream magnetic spectrometer with a sci-fiber tracker. A cooling cell will then follow, alternating energy loss in absorbers and RF acceleration. A second spectrometer identical to the first and a second muon identification system measure the outgoing emittance. In the 2010 run the beam line with various detectors has been commissioned, and measurements of the particle rate and the emittance of the beam with time-of-flight detectors is reported.

The 2011 Europhysics Conference on High Energy Physics-HEP 2011,

July 21-27, 2011

Grenoble, Rhône-Alpes France

* Speaker.

${ }^{\dagger}$ Website: mice.iit.edu 


\section{Introduction}

MICE is proof-of-principle experiment designed to demonstrate the technique of muon ionisation cooling for the first time. Ionisation cooling is a neccessary technology for any future facility based on intense stored muon beams. Such facilities include a Neutrino Factory, the most powerful tool for studying leptonic CP violation, and a Muon Collider, which offers a potential route to multi-TeV lepton - anti-lepton collisions [1]. In particular, MICE is designed to deliver an input muon beam of variable emittance in the range $140 \mathrm{MeV} / \mathrm{c}$ to $240 \mathrm{MeV} / \mathrm{c}$, reduce the emittance by $\sim 10 \%$, and measure this reduction to within $1 \%$ (absolute emittance measurements of $0.1 \%$ ).

\section{The Beam line}

MICE is hosted by the Rutherford Appleton Laboratory, UK, using the $800 \mathrm{MeV}$ ISIS synchrotron as a proton driver. A schematic of the MICE beam line is shown in Fig. 1. A titanium target oscillates into the circulating proton beam generating a pion shower. Part of this shower is captured by a quadrupole triplet, and is then transported to a $5 \mathrm{~T}$ superconducting decay solenoid. Two dipole magnets are used to redirect the beam and perform momentum selection on the particles, followed by two further quadrupole triplets prior to the beam stop. Various particle identification detectors are positioned along the beam line including a scintillator counter, two aerogel Cherenkov detectors and three 50 - 60 ps resolution time-of-flight (TOF) stations. Each TOF station is composed of two perpendicular scintillator bar layers, each bar being readout at either end by a photomultiplier tube [2].

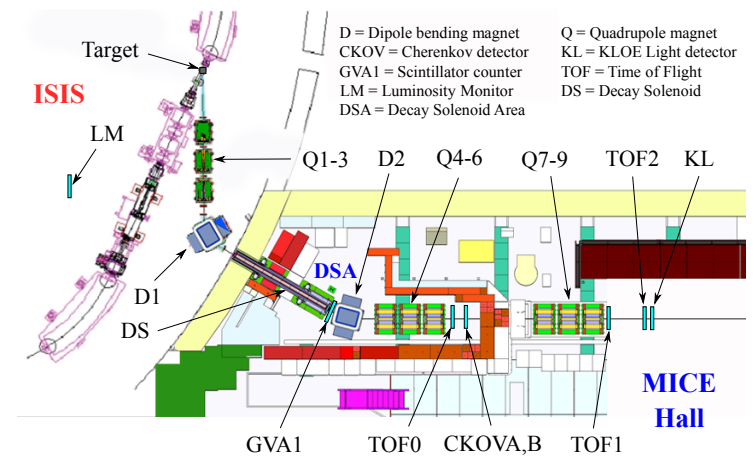

Figure 1: The MICE Beam line. The cooling channel will be placed between TOF1 and TOF2.

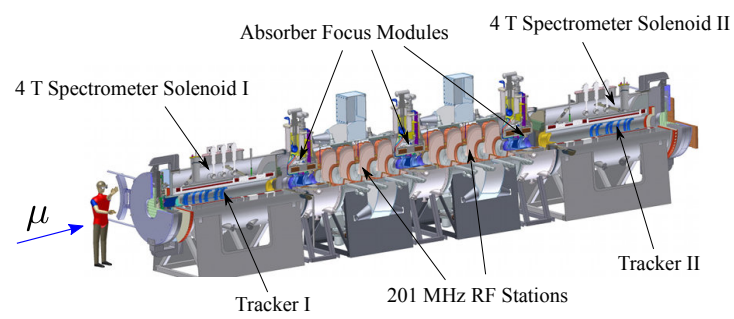

Figure 2: A schematic of the MICE cooling channel.

\section{The Cooling Channel}

A schematic of the MICE cooling channel is shown in Fig. 2. It consists of three absorber modules interspersed by two radio frequency (RF) cavities. The absorber modules reduce particle momentum in all three spatial directions, as particles ionise the medium and lose energy. The RF cavities then resupply the lost energy in the longitudinal direction only, leading to a reduction in transverse emittance. Two high precision scintillating fibre trackers, placed in $4 \mathrm{~T}$ superconducting solenoids, measure the emittance before and after cooling [3]. A lead diffuser is placed immediately 
upstream of the first tracker, in order to inflate the beam emittance prior to cooling. The production of the cooling channel is only partially complete, with expected delivery of the trackers and first absorber module in the second half of 2012, and the second absorber and first RF cavity in the first half of 2014.

\section{Particle Rates and Beam Loss}

The particle rate in the MICE beam line may be measured using the TOF stations. The TOF stations may also be used to perform PID by measuring the TOF between stations, allowing the determination of the number of muon tracks successfully reconstructed between any two stations. An example TOF spectrum between TOF0 and TOF1, for a beam line optimised for pion to muon transport (as will be used for the cooling measurement), is shown in Fig. 3. A large muon peak can be seen on the right, which contains a small amount of pions, estimated to be of order $\sim 1 \%$ from simulation.

In addition to providing particles for the MICE beam line, the action of the MICE target also has the undesirable secondary effect of increasing beam loss levels in the host accelerator. This is measured by use of 39 argon wire ionisation chambers positioned around the inside of the ISIS ring. ISIS is split in to 10 repeating super periods, SP0 - SP9, with the MICE target being positioned at the start of SP7. A figure of merit, used to quantify beam loss due specifically to the action of the MICE target, may be defined as the beam losses recorded in the four SP7 beam loss monitors, integrated over the $10 \mathrm{~ms}$ of the ISIS injection - extraction cycle.

The particle rate and beam loss may be correlated in order to produce plots of rate per unit beam loss. Results of such a study performed in June 2010 are shown in Fig. 4 for a beam line set for negative pion to muon transport, using a $3.2 \mathrm{~ms}$ capture gate. A clear linear relationship between beam loss and particle rate can be seen.

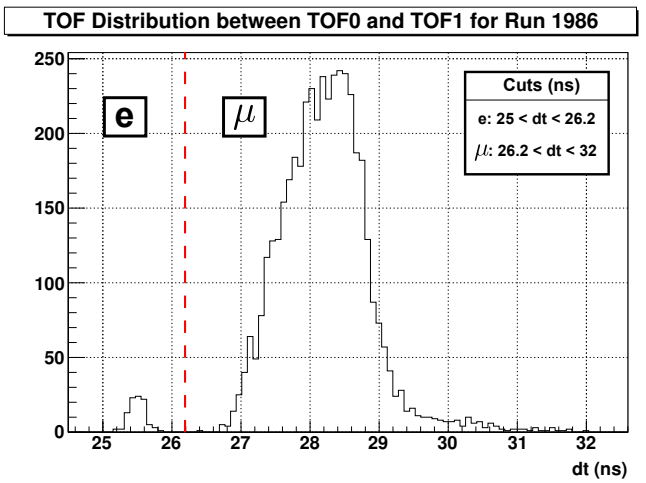

Figure 3: TOF distribution of particles between TOF0 and TOF1 for a beam line optimised for negative $\pi \rightarrow \mu$ transport (15/06/10 study).

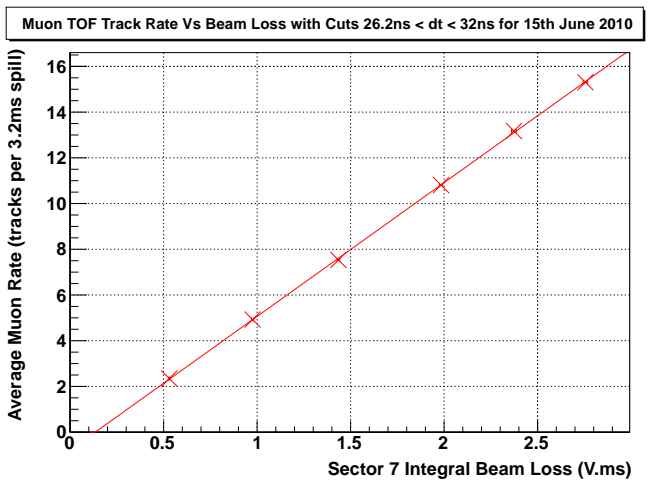

Figure 4: Reconstructed muon track rate between TOF0 and TOF1, as a function of SP7 beam loss in ISIS. A negative $\pi \rightarrow \mu$ beam line is used, with rates captured in a $3.2 \mathrm{~ms}$ gate $(15 / 06 / 10$ study).

\section{Beam Emittance and Optical Parameters}

The full MICE emittance measurement is to be performed using the high precision sci-fibre 
trackers mentioned earlier. While these remain in commissioning however, a novel approach to emittance measurement using the TOF stations has been performed. A detailed description of this technique can be found in [4]; a brief summary is presented here.

Using the arrival time of pulses at the PMTs of the scintilltor bars, each TOF station may be used to provide $(x, y)$ position information for the beam with a resolution of $\sim 1 \mathrm{~cm}$ in each direction, in addition to the timing information, $t . \quad(x, y, t)$ are found at TOF0 and TOF1, and TOF used to identify which tracks are from muons. An initial guess is made of the longitudinal momentum, $p_{z}$, and momentum-dependent transport matrices are then used to map particle motion from one station to the other. This provides an estimate of the path length, which in turn can be used to provide an improved estimate of $p_{z}$. This momentum estimate is then fed back into the transport matrices, leading to the elimination of the initial path length bias. This procedure proceeds iteratively, converging after five iterations. The correct value of $p_{z}$ then gives accurate transport matrices, which when combined with the position measurements at both TOF0 and TOF1 allow the determination of the angles of the particle motion from the horizontal, $x^{\prime}$ and $y^{\prime}$. This then provides the trace space $\left(x, x^{\prime}\right),\left(y, y^{\prime}\right)$ and the beam emittance.

Example results of applying this procedure are shown in Fig. 5. Monte Carlo (MC) truth is shown in $(a)$, resolution smeared MC reconstruction in $(b)$ and real data in $(c)$. These distributions may be parameterised with trace space ellipses, with the area corresponding to the beam emittance, and the orientation being determined by the Twiss parameters. After a cut to remove difficult to model high amplitude muons, good agreement is found between reconstructed MC and data.
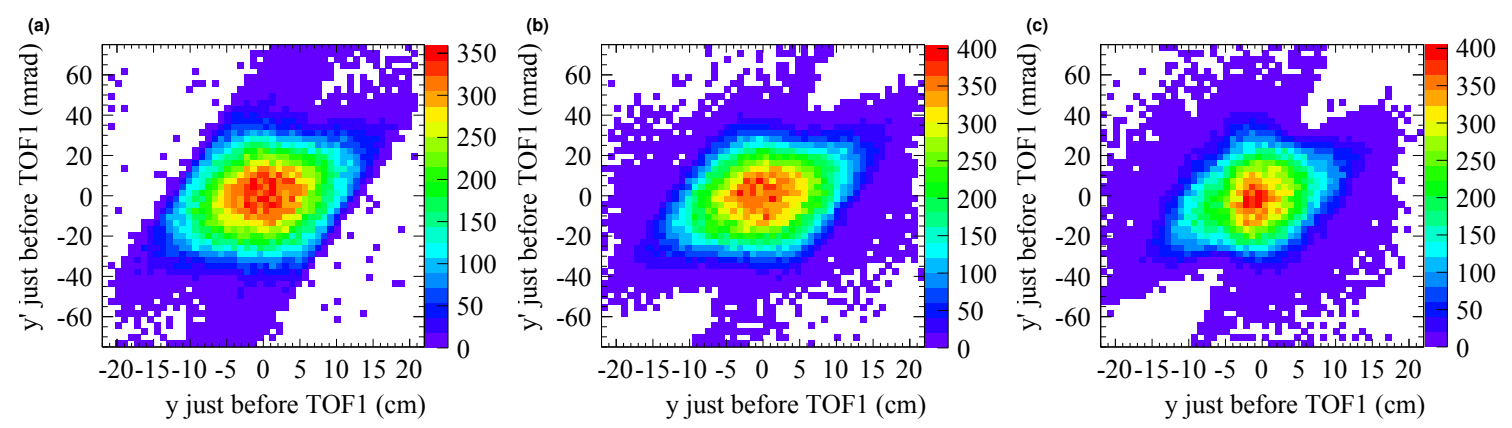

Figure 5: MICE emittance plots for a (6 mm, $200 \mathrm{MeV} / \mathrm{c})$ negative muon beam. (a) shows MC truth, (b) resolution smeared reconstructed $\mathrm{MC}$ and (c) real data.

\section{References}

[1] S. Geer. Muon Colliders and Neutrino Factories. Annl. Rev. Nucl. Part. Sci., 59:347-365, 2009.

[2] R. Bertoni et al. The Design and Commissioning of the MICE Upstream Time-of-Flight System. Nucl. Instr. and Meth., A615:14-26, 2010.

[3] M. Ellis et al. The Design, Construction and Performance of the MICE Scintillating Fibre Trackers. arXiv:1005.3491v2, 2010.

[4] M. A. Rayner. The Development of a Novel Technique for Characterizing the MICE Muon Beam and Demonstrating its Suitability for a Muon Cooling Measurement. PhD Thesis, The University of Oxford, 2011. 